\title{
Markers of insulin resistance are associated with fatness and fitness in school-aged children: the European Youth Heart Study
}

\author{
J. R. Ruiz • N. S. Rizzo • F. B. Ortega • H. M. Loit • \\ T. Veidebaum • M. Sjöström
}

Received: 21 December 2006 / Accepted: 22 March 2007 /Published online: 11 May 2007

(C) Springer-Verlag 2007

\begin{abstract}
Aims/hypothesis Our aim was to examine the association between markers of insulin resistance and: (1) body fat and waist circumference, taking into account cardiorespiratory fitness in school-aged children; and (2) cardiorespiratory fitness at differing levels of body fat and waist circumference. Subjects and methods This was a cross-sectional study of 873 children aged 9.6 \pm 0.4 years from Estonia and Sweden. Weight, height and waist circumference were measured. Body fat was expressed as the sum of five skinfold thicknesses. Cardiorespiratory fitness was estimated by a maximal cycle-ergometer test. The studied markers of insulin resistance were fasting insulin and glucose, and homeostasis model assessment (HOMA).

Results HOMA and fasting insulin were positively associated with body fat and waist circumference after adjusting for cardiorespiratory fitness, age, pubertal status and study location. HOMA and fasting insulin were negatively associated with cardiorespiratory fitness in children in the third (highest) tertile of body fat and waist circumference after controlling for sex, age, pubertal status and study location. Fasting glucose was negatively associated with
\end{abstract}

J. R. Ruiz $(\bowtie) \cdot$ N. S. Rizzo • F. B. Ortega $\cdot$ M. Sjöström

Unit for Preventive Nutrition,

Department of Biosciences and Nutrition, NOVUM,

Karolinska Institutet,

14157 Huddinge, Sweden

e-mail: ruizj@ugr.es

J. R. Ruiz $\cdot$ F. B. Ortega

Department of Physiology, School of Medicine,

University of Granada,

Granada, Spain

H. M. Loit • T. Veidebaum

Estonian Centre of Behavioural and Health Sciences,

Tallinn, Estonia cardiorespiratory fitness in children in the third (highest) tertile of waist circumference, but it was not associated when body fat was taken into account.

Conclusions/interpretation In school-aged children, HOMA and fasting insulin are significantly associated with body fat and waist circumference. In addition, cardiorespiratory fitness explains a significant proportion of the HOMA and fasting insulin variance in those children with high levels of body fat and waist circumference. The findings suggest that the deleterious consequences ascribed to high fatness could be counteracted by having high levels of cardiorespiratory fitness.

Keywords Body fat . Cardiorespiratory fitness - Children . Diabetes · Glucose · HOMA · Insulin resistance · Obesity · Physical activity
Abbreviations
EYHS European Youth Heart Study
HOMA homeostasis model assessment

\section{Introduction}

One of the consequences of the paediatric obesity epidemic is thought to be an increased incidence of type 2 diabetes among children worldwide [1]. Longitudinal studies have shown that childhood obesity is closely related to markers of insulin resistance later in life [2]. Both total and central adiposity are also strongly negatively associated with cardiorespiratory fitness in young people [3-5]. Therefore, the detrimental consequences attributed to adiposity may be partially due to the influence of low cardiorespiratory fitness.

Findings from cross-sectional and prospective studies show that markers of insulin resistance and other cardio- 
vascular disease risk factors are already negatively associated with high cardiorespiratory fitness in adolescents [68]. However, studies examining the interaction of markers of insulin resistance and fatness and fitness in a large sample of school-aged children are sparse and equivocal [9, 10]. The purpose of the present study was to examine: (1) the association between markers of insulin resistance and body fat and waist circumference, taking into account cardiorespiratory fitness; and (2) to examine the association of markers of insulin resistance with cardiorespiratory fitness at differing levels of body fat and waist circumference in school-aged children.

\section{Subjects and methods}

The study involved 1,140 children aged 9.6 \pm 0.4 years from the Estonian and Swedish parts of the European Youth Heart Study (EYHS). A complete data set was available in 873 children ( 444 girls and 429 boys), except for pubertal status, which was available in 672 children (77\%). None of the analysed variables differed significantly between the included children $(n=873)$ and those who did not provide a whole set of data $(n=1,140)$. The EYHS is a school-based cross-sectional study designed to examine the interactions between personal, environmental and lifestyle influences on the risk factor for future cardiovascular diseases. Study design, selection criteria and sample calculations have been reported elsewhere [11, 12]. Data collection took place during the school year 1998-1999. In Estonia, the city of Tartu and its surrounding rural region was the geographical sampling area. In Sweden, eight municipalities (Botkyrka, Haninge, Huddinge, Nynäshamn, Salem, Södertälje, Tyresö and Örebro) were chosen for data collection. All schools with more than 20 children in the age group in question were initially included. The schools were stratified with regard to school grade and to the mean income level in their catchment areas (below or above the mean in their municipality). A random sampling procedure from each stratum was carried out, and the sampled schools were invited to participate in the study. From the complete lists of pupils in the collaborating schools, groups of pupils proportional to the sizes of the respective schools were randomly selected. The local ethical committees approved the study (Örebro City Council no. 690/98, Huddinge University Hospital no. 474/98 and University of Tartu no. 49/30-1997). One parent or legal guardian provided written informed consent, and all children gave verbal consent.

Physical examination Height and weight were measured by standardised procedures. BMI was calculated as weight/height squared $\left(\mathrm{kg} / \mathrm{m}^{2}\right)$. Skinfold thicknesses were measured with a Harpenden calliper at the biceps, triceps, subscapular, supra- iliac and triceps surae areas on the left side of the body according to the criteria described elsewhere [13]. All measurements were taken twice and in rotation, and the mean value was calculated. If the difference between the measurements was $>2 \mathrm{~mm}$, a third measurement was taken and the two closest measurements were averaged. The sum of the five skinfold thicknesses (hereafter referred as 'body fat') rather than BMI was used as an indicator of total body fat, because BMI has been suggested to be a less valid measurement of body fatness in children [14], and because BMI does not discriminate between muscle and fat mass. Waist circumference $(\mathrm{cm})$ was measured in duplicate with a metal anthropometric tape midway between the lowest rib and the iliac crest at the end of a gentle expiration. The mean between two measurements was used for further calculations. If the two measurements differed by $>1 \mathrm{~cm}$, a third measurement was taken and the two closest measurements were averaged.

Identification of pubertal development was also assessed [15]. Pubertal stage was recorded by a researcher of the same sex as the child, after brief observation. Breast development in girls and genital development in boys was used for pubertal classification.

Blood samples Serum concentrations of glucose and insulin were measured after an overnight fast. The homeostasis model assessment (HOMA) was calculated [16] as fasting insulin $(\mathrm{mU} / \mathrm{l}) \times$ fasting glucose $(\mathrm{mmol} / \mathrm{l}) / 22.5$ (to convert insulin values in $\mathrm{mU} / \mathrm{l}$ to $\mathrm{pmol} / \mathrm{l}$ multiply by 6.945 ). A detailed description of the blood analysis has been reported elsewhere [17].

Cardiorespiratory fitness Cardiorespiratory fitness was determined by a maximum cycle-ergometer test [18]. Briefly, the workload was pre-programmed on a computerised cycleergometer (Monark 829E; Ergomedic, Vansbro, Sweden) to increase every third minute until exhaustion. Criteria for exhaustion were a heart rate $\geq 185$ beats per min, failure to maintain a pedalling frequency of at least 30 revolutions per min, and a subjective judgement by the observer that the child could no longer keep up, even after vocal encouragement. The power output was calculated as $W_{1}+\left(W_{2} \times t / 180\right)$, where $W_{1}$ is the work rate at the fully completed stage, $W_{2}$ is the work rate increment at the final incomplete stage, and $t$ is time in seconds at the final incomplete stage. Cardiorespiratory fitness was expressed as the maximal power output per $\mathrm{kg}$ of body mass $(W / \mathrm{kg})$. The test used to measure cardiorespiratory fitness has been previously validated in children of the same age [19].

Statistical analysis The data are presented as means $\pm \mathrm{SD}$ unless otherwise stated. To achieve normality in the residuals, waist circumference, skinfold thickness, insulin 
and glucose were transformed to the natural logarithm, and HOMA was raised to the power of $1 / 3$. Sex differences were assessed by one-way ANOVA, and adjusted for mass significance [20].

The association between markers of insulin resistance and body fat and waist circumference was assessed by oneway analysis of covariance with waist circumference or body fat variables as fixed factors, HOMA, insulin or glucose as dependent variables, and cardiorespiratory fitness, age, pubertal status and study location as covariates. Since there was an interaction effect between sex and either body fat or waist circumference, all analyses were performed separately for boys and girls. Both body fat and waist circumference were recoded into tertiles to be entered into the models. In girls, mean body fat values for the first, second and third tertiles were 26.8, 36.3 and $59.4 \mathrm{~mm}$, respectively. In boys, mean body fat values for the first, second and third tertiles were 25.9, 35.2 and $57.6 \mathrm{~mm}$, respectively. In girls, mean waist circumference values for the first, second and third tertiles were 53.4, 58.7 and $65.5 \mathrm{~cm}$, respectively. In boys, mean waist circumference values for the first, second and third tertiles were 53.6, 57.2 and $61.7 \mathrm{~cm}$, respectively. For those relationships where waist circumference was involved, an additional adjustment for height or body fat was done.

Following bivariate correlation analysis, multiple regressions were used to study the association between markers of insulin resistance and cardiorespiratory fitness after controlling for sex, age, pubertal status and study location. Regression analysis was performed separately by body fat and waist circumference tertiles (low, middle and high, equals first, second and third tertile, respectively). No interaction effects between sex and cardiorespiratory fitness was found; therefore, all analyses were performed jointly for boys and girls. Semi-partial correlation was used as the measure of the relationship between cardiorespiratory fitness and the part of the outcome (HOMA, fasting insulin and glucose) that is not explained by the other predictors in the model (sex, age, pubertal status and study location). In other words, it is a measure of the variance in the outcome that fitness alone shares. The analyses were performed using the Statistical Package for Social Sciences (SPSS, v. 14.0 for Windows; SPSS Inc., Chicago, IL) and the level of significance was set to 0.05 .

\section{Results}

The descriptive characteristics of the study sample are shown in Table 1. Girls had significantly lower waist circumferences, glucose levels and fitness levels than boys, while boys had lower body fat, HOMA and insulin than girls. The Estonian children (both girls and boys) had lower body fat and waist circumference than did the Swedish children, whereas the former had higher HOMA and insulin levels.

All the fatness-related parameters were positively intercorrelated in both girls and boys, with correlation coefficients ranging from 0.751 to 0.844 . Cardiorespiratory fitness was significantly associated with body fat, BMI and waist circumference in girls $(r=-0.437,-0.407$ and -0.312 , respectively, all $p<0.001)$ and boys $(r=-0.562,-0.487$ and -0.439 , respectively, all $p<0.001$ ).

The associations of HOMA, fasting insulin and glucose with body fat and waist circumference for girls and boys are shown in Fig. 1. HOMA and fasting insulin were positively associated (all $p<0.001$ ) with body fat and waist circumference in both girls and boys (Fig. 1). Fasting glucose was not significantly associated with body fat or waist circumference (Fig. 1). For those relationships where waist

Table 1 Descriptive characteristics of the children

\begin{tabular}{|c|c|c|c|c|}
\hline & All $(n=873)$ & Girls $(n=444)$ & Boys $(n=429)$ & $p$ value for sex \\
\hline Age (years) & $9.6 \pm 0.4$ & $9.5 \pm 0.4$ & $9.6 \pm 0.4$ & 0.147 \\
\hline Pubertal status (I/II/III/IV/V) ${ }^{\mathrm{a}}$ & $80 / 18 / 2 / 0 / 0$ & $70 / 26 / 4 / 0 / 0$ & $90 / 10 / 0 / 0 / 0$ & - \\
\hline Weight $(\mathrm{kg})$ & $32.1 \pm 5.8$ & $32.0 \pm 6.2$ & $32.1 \pm 5.5$ & 0.828 \\
\hline Height (m) & $1.4 \pm 0.1$ & $1.4 \pm 0.1$ & $1.4 \pm 0.1$ & 0.677 \\
\hline BMI $\left(\mathrm{kg} / \mathrm{m}^{2}\right)$ & $16.7 \pm 2.1$ & $16.7 \pm 2.3$ & $16.8 \pm 2.0$ & 0.846 \\
\hline Body fat (mm skinfold thickness) ${ }^{b}$ & $40.2 \pm 16.9$ & $44.6 \pm 18.2$ & $35.7 \pm 14.2$ & $<0.001$ \\
\hline Waist circumference $(\mathrm{cm})^{\mathrm{b}}$ & $58.0 \pm 5.4$ & $57.1 \pm 5.9$ & $58.9 \pm 4.8$ & $<0.001$ \\
\hline $\mathrm{HOMA}^{\mathrm{c}}$ & $1.3 \pm 0.8$ & $1.4 \pm 0.8$ & $1.2 \pm 0.8$ & $<0.001$ \\
\hline Insulin $(\mathrm{mU} / \mathrm{l})^{\mathrm{b}, \mathrm{d}}$ & $41.4 \pm 23.5$ & $44.7 \pm 24.5$ & $38.0 \pm 21.9$ & $<0.001$ \\
\hline Glucose $(\mathrm{mmol} / \mathrm{l})^{\mathrm{b}}$ & $5.0 \pm 0.4$ & $4.8 \pm 0.4$ & $5.1 \pm 0.3$ & $<0.001$ \\
\hline Cardiorespiratory fitness $(W / \mathrm{kg})$ & $2.9 \pm 0.5$ & $2.7 \pm 0.4$ & $3.2 \pm 0.5$ & $<0.001$ \\
\hline
\end{tabular}

All values are means $\pm \mathrm{SD}$, or ${ }^{\mathrm{a}}$ percentages

Non-transformed data are presented in this table, but analyses (Table 2 and Figs. 1 and 2) were performed on

${ }^{b} \log$-transformed data or ${ }^{\mathrm{c}}$ data transformed to the power of $1 / 3$

${ }^{\mathrm{d}}$ To convert insulin values in $\mathrm{mU} / 1$ to pmol/1 multiply by 6.945 . 
Fig. 1 Markers of insulin resistance stratified by body fat (expressed as skinfold thickness) and waist circumference tertiles in girls and boys. a HOMA and body fat tertiles, $\mathbf{b}$ insulin and body fat tertiles, $\mathbf{c}$ glucose and body fat tertiles, d HOMA and waist circumference tertiles, e insulin and waist circumference tertiles, f glucose and waist circumference tertiles. Data were analysed by one-way analysis of covariance after adjusting for cardiorespiratory fitness, age, pubertal status and study location. White bars, low (first) tertiles; grey bars, middle (second) tertiles; black bars, high (third) tertiles. Body fat, waist circumference, and fasting plasma insulin and glucose were transformed to the natural logarithmic, and HOMAs were raised to the power of $1 / 3$. HOMA and fasting insulin were positively associated (all $p<0.001$ ) with body fat and waist circumference in both girls and boys. Fasting glucose was not significantly associated with body fat or waist circumference. Values are means, and errors bars represent $95 \%$ confidence intervals. To convert plasma insulin values in $\mathrm{mU} / 1$ to $\mathrm{pmol} / \mathrm{l}$ multiply by 6.945
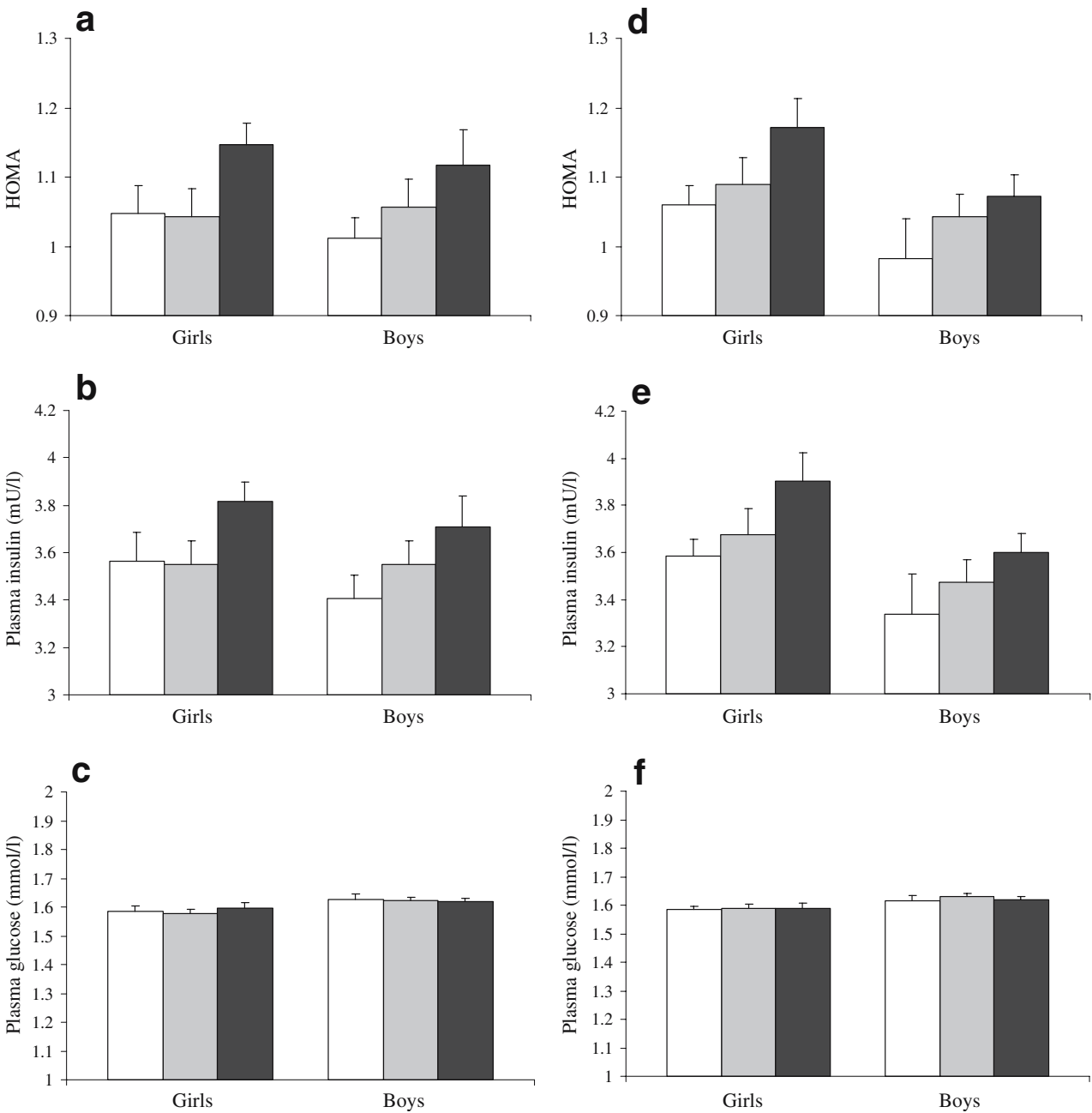

circumference was involved, an additional adjustment for height or body fat did not alter the results.

Figure 2 shows simple bivariate relationships between HOMA and cardiorespiratory fitness by body fat and waist circumference tertiles for girls and boys. The results of the regression models with HOMA, fasting insulin and glucose as the outcome variables and cardiorespiratory fitness as the predictor variable, conducted by body fat and waist circumference tertiles, are shown in Table 2. HOMA and fasting insulin were negatively associated with cardiorespiratory fitness in those children with high levels of body fat and waist circumference (those in the third tertile). Subsequent analysis examining the association of markers of insulin resistance with cardiorespiratory fitness separately by BMI tertiles was similar to those with body fat. Fasting glucose was negatively associated with cardiorespiratory fitness in children in the third (highest) tertile of waist circumference, but it was not associated when body fat was taken into account. For the other relationships where waist circumference was involved, additionally controlling for height or body fat did not alter the results.
Similar results were obtained when the regression analyses were performed separately for girls and boys. No significant effect of pubertal status on the outcome was observed in any of the analysis performed (data not shown).

\section{Discussion}

The results of the present study showed that HOMA and fasting insulin were positively associated with body fat and waist circumference in school-aged children. In addition, cardiorespiratory fitness explained a significant proportion of the HOMA and fasting insulin variance in those children with high levels of body fat and waist circumference.

The methodology used allowed us to examine the association of markers of insulin resistance with fitness within different levels of total and central fatness (i.e. low, middle and high). The findings suggested that the deleterious consequences ascribed to high fatness may be counteracted by having high levels of cardiorespiratory fitness. Taken together, these findings suggest that inter- 

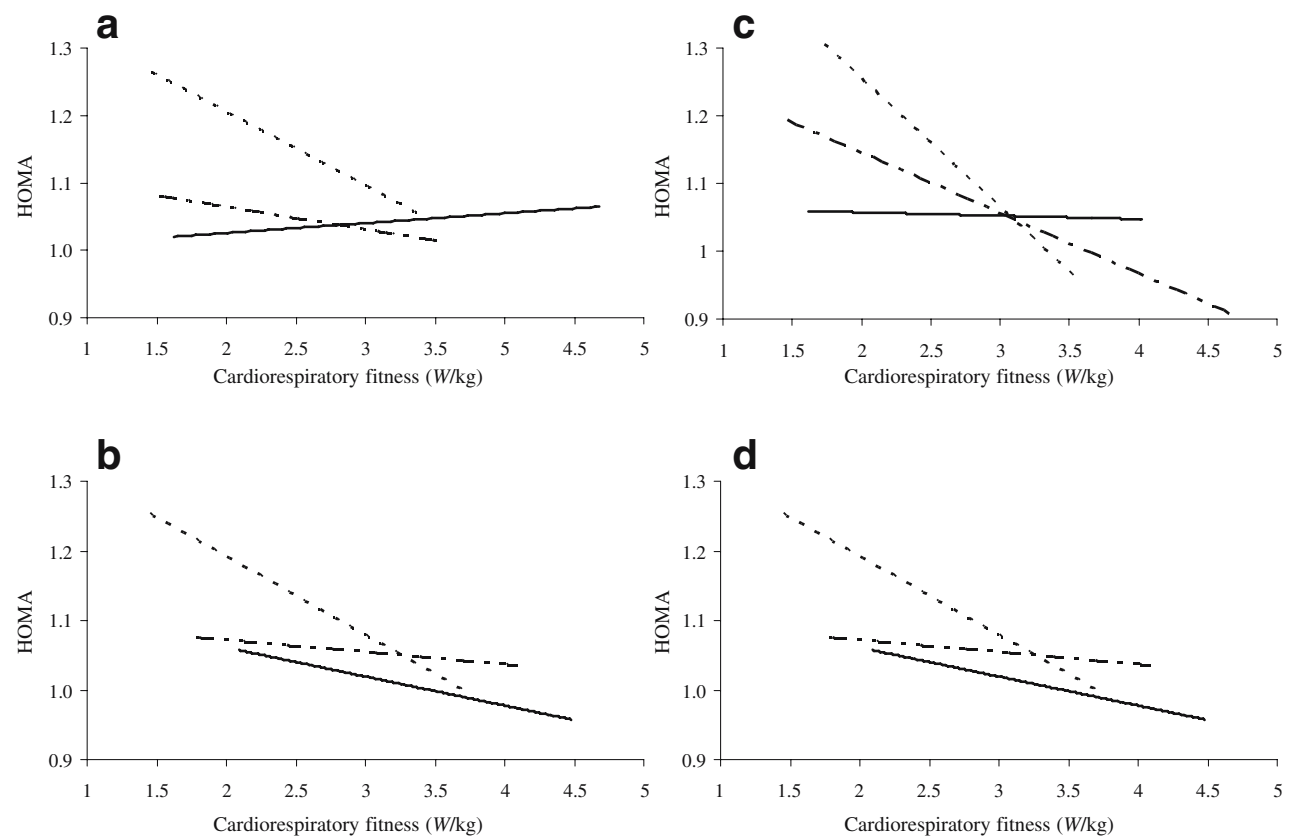

Fig. 2 Relationship between HOMA and cardiorespiratory fitness by body fat $(\mathrm{BF})(\mathbf{a}, \mathbf{b})$ and waist circumference $(\mathrm{WC})(\mathbf{c}, \mathbf{d})$ tertiles for girls $(\mathbf{a}, \mathbf{c})$ and boys $(\mathbf{b}, \mathbf{d})$ boys. a, b Solid line, low body fat first tertile); dot/dash line, middle body fat (second tertile); dashed line, high body fat (third tertile). c, d Solid line, low waist circumference (first tertile); dot/dash line, middle waist circumference (second tertile); dashed line, high waist circumference (third tertile). HOMA

data were raised to the power of $1 / 3$ before analysis. Girls: low BF, $r=0.040(p=0.703)$; middle $\mathrm{BF}, r=-0.062(p=0.456)$; high $\mathrm{BF}, r=-0.218$ $(p=0.002)$; low WC, $r=-0.011$ ( $p=0.862)$; middle WC, $r=-0.221 \quad(p=0.012)$; high WC, $r=-0.407(p<0.001)$. Boys: low BF, $\mathrm{r}=-0.093 \quad(p=0.197)$; middle BF, $r=-0.042(p=0.623)$; high BF, $r=-0.252(p=0.016)$; low WC, $r=-0.312(p=0.018)$; middle WC, $r=-0.167(p=0.032)$; high WC, $r=-0.197(p=0.004)$

ventions designed to prevent hyperinsulinaemia and related metabolic disorders should focus not only on reducing fatness but also improving fitness.

Numerous studies support a link between metabolic risk factors and adiposity in children [6, 21]. Fasting insulin appears to be related more to total adiposity than to central adiposity $[6,22]$. However, one study in youths found that total body fat was the predominant factor influencing insulin sensitivity, but that visceral fat may have additional effects on fasting insulin [23]. In our study, both body fat (as a marker of total adiposity) and waist circumference (as a marker of central adiposity) explained a significant proportion of the HOMA and fasting insulin variance after adjusting for potential confounders including cardiorespiratory fitness. Waist circumference has been shown to be a powerful marker of abdominal fat accumulation and visceral adiposity tissue in young people [24, 25]. Waist circumference is widely used as a surrogate of central fat distribution in young and adult people [25]. Direct measurements of visceral adiposity with magnetic resonance imaging or computed tomography may provide further information; however, this can not be proposed for field studies due to their cost and technical difficulties [26].

The results of the association between markers of insulin resistance and cardiorespiratory fitness separately by BMI tertiles were similar to those with body fat. Limitations of

BMI are well recognised, as well as its feasibility of being used in epidemiological studies. Recently, a cross-sectional study on 407 children aged 7-16 years reported that BMI was a good predictor of the variance of subcutaneous adipose tissue (88.9\% of variance) [25]. Therefore, when no better possibilities are available, BMI could be used as a marker of subcutaneous adipose tissue in children.

The association between fasting insulin and cardiorespiratory fitness is consistent with findings reported in North American adolescents [6, 7], whereas other studies failed to find associations between markers of insulin resistance and cardiorespiratory fitness $[9,10]$. Gutin et al. [6] reported that the percentage of body fat (measured with dual-energy $\mathrm{X}$-ray absorptiometry) and cardiorespiratory fitness explained a significant proportion of the fasting insulin variance in boys. In girls, fasting insulin was associated only with percentage of body fat, suggesting that the detrimental impact of high fatness and low fitness was greater in boys than in girls. In our study, cardiorespiratory fitness explained a significant proportion of the HOMA and fasting insulin variance in both girls and boys with high levels of fatness. The North American adolescents were both older (14-18 years) and biologically more mature (Tanner stages 4-5) than the children involved in our study. Rapid and dynamic changes in various metabolic systems, including hormonal regulation, changes in body fat content 
Table 2 Multiple regression coefficients $(\beta)$, coefficient of determination $\left(R^{2}\right)$, and semipartial correlations $(s r)$ examining the association of markers of insulin resistance with cardiorespiratory fitness separately by body fat and waist circumference tertiles

All the analyses were controlled for sex, age, pubertal status and study location. Analysis was performed on ${ }^{a}$ data raised to the power of $1 / 3$ or ${ }^{\mathrm{b}} \log$-transformed data. Low, middle and high indicate first, second and third tertile, respectively

\begin{tabular}{|c|c|c|c|c|c|}
\hline Outcome variable & Cardiorespiratory fitness & $\beta$ & $p$ value & $R^{2}$ & $s r$ \\
\hline \multicolumn{6}{|l|}{ By body fat tertiles } \\
\hline \multirow[t]{3}{*}{ HOMA $^{\mathrm{a}}$} & Low fat & 0.095 & 0.232 & 0.087 & 0.08 \\
\hline & Middle fat & -0.009 & 0.909 & 0.079 & -0.08 \\
\hline & High fat & -0.241 & $>0.001$ & 0.156 & -0.23 \\
\hline \multirow[t]{3}{*}{ Insulin $^{\text {b }}$} & Low fat & 0.108 & 0.174 & 0.088 & 0.09 \\
\hline & Middle fat & -0.005 & 0.948 & 0.065 & -0.01 \\
\hline & High fat & -0.220 & 0.002 & 0.149 & -0.21 \\
\hline \multirow[t]{3}{*}{ Glucose $^{\mathrm{b}}$} & Low fat & -0.084 & 0.283 & 0.126 & -0.07 \\
\hline & Middle fat & -0.010 & 0.899 & 0.132 & -0.01 \\
\hline & High fat & -0.126 & 0.081 & 0.067 & -0.12 \\
\hline \multicolumn{6}{|c|}{ By waist circumference tertiles } \\
\hline \multirow[t]{3}{*}{$\mathrm{HOMA}^{\mathrm{a}}$} & Low circumference & -0.010 & 0.905 & 0.100 & -0.01 \\
\hline & Middle circumference & -0.148 & 0.063 & 0.086 & -0.13 \\
\hline & High circumference & -0.250 & 0.001 & 0.215 & -0.22 \\
\hline \multirow[t]{3}{*}{ Insulin $^{\mathrm{b}}$} & Low circumference & 0.009 & 0.911 & 0.099 & 0.01 \\
\hline & Middle circumference & -0.139 & 0.069 & 0.110 & -0.13 \\
\hline & High circumference & -0.223 & 0.002 & 0.183 & -0.20 \\
\hline \multirow[t]{3}{*}{ Glucose $^{\mathrm{b}}$} & Low circumference & -0.056 & 0.492 & 0.056 & -0.05 \\
\hline & Middle circumference & 0.002 & 0.977 & 0.152 & 0.01 \\
\hline & High circumference & -0.183 & 0.010 & 0.104 & -0.16 \\
\hline
\end{tabular}

and body fat distribution, as well as transient changes in insulin resistance, are known to occur during growth and puberty [1], which may partially explain the differences between studies.

Results from the Study of Latino Adolescents at Risk for Diabetes revealed that insulin sensitivity or secretion was not independently associated with cardiorespiratory fitness in a relatively small sample of overweight Hispanic children aged 8-13 years with a family history of type 2 diabetes [9]. Moreover, cardiorespiratory fitness was not shown to be different in children with impaired glucose tolerance compared with those with normal glucose tolerance [10], suggesting that fitness may influence insulin dynamics indirectly through fat mass, especially in those groups of children at increased risk of early cardiovascular disease. Differences between study subjects and methodologies make comparisons difficult. Moreover, it must be borne in mind that the children involved in our study were apparently healthy children with no previously diagnosed cardiovascular pathologies.

Recent findings from the National Health and Nutrition Examination Survey [7] showed that insulin sensitivity was significantly associated with physical activity (measured by questionnaires) and cardiorespiratory fitness in boys. In girls, insulin sensitivity was not significantly associated with physical activity or with cardiorespiratory fitness but was significantly associated with BMI. The differences between sexes may be due to the fact that females seem to be, in some way, protected from the prejudicial effects of excess fatness by the role of oestrogens [27, 28]. Similarly, this mechanism also seems to protect young women from the deleterious consequences of low cardiorespiratory fitness on insulin resistance. This might be true due to the fact that fitness levels are lower in girls than in boys, whereas fatness and fasting insulin levels are higher in the former. This is consistent with other studies on young people [6, 7, 29-32].

Cardiorespiratory fitness is highly influenced by the person's activity level [3, 4, 33, 34]. Objectively measured physical activity has also been negatively associated with body fat activity level [3, 4], and with fasting insulin levels [35]. Similarly, HOMA was positively associated with regular exercise independently of total and central adiposity in adolescents [36]. Collectively, these findings indicate that regular physical activity may play a key role in reducing fat mass and markers of insulin resistance and improving cardiorespiratory fitness in youths.

In the present study fasting glucose was not associated with fatness and fitness. Similarly, fasting glucose was not associated with total physical activity in school-aged Danish girls and boys [35]. In fact, the clinical value of fasting glucose in the first decade of life has been questioned. Recent findings show that fasting glucose is weakly associated with clustering of cardiovascular disease risk factors in young people [37]. This can be explained by the fact the insulin resistance may increase before children display impaired glucose tolerance. In the very early stages of insulin resistance, the pancreas may compensate for this metabolic disturbance by excreting higher quantities of insulin [38].

The limitations of the present study include its crosssectional design; therefore, the direction of causality can not be determined. The HOMA model is a method for 
assessing beta cell function and insulin resistance from fasting insulin and glucose concentrations, whereas the gold standard for measuring insulin sensitivity/resistance is the euglycaemic-hyperinsulinaemic clamp. However, the latter method is too invasive and costly, and may not be suitable for large epidemiological studies. HOMA has been compared with a number of well-validated methods used to measure insulin resistance and beta cell function [39].

In conclusion, the results showed that HOMA and fasting insulin were associated with body fat and waist circumference in school-aged children. In addition, cardiorespiratory fitness explained a significant proportion of the HOMA and fasting insulin variance in those children with high levels of body fat and waist circumference. Collectively, the findings suggest that the deleterious consequences ascribed to high fatness could be counteracted by having high levels of cardiorespiratory fitness. The assessment of the effect of an exercise intervention focused on reducing fatness and improving fitness is warranted.

Acknowledgements We are indebted to late M. Harro for her enthusiasm and foresight in her scientific work during her period as Principal Investigator for the Estonian part of the European Youth Heart Study. We thank O. Carlsson from the Unit for Preventive Nutrition, Department of Biosciences and Nutrition at NOVUM, Karolinska Institutet, for statistical assistance. The Swedish part of the study was supported by grants from the Stockholm County Council (M. Sjöström), and the Estonian part was supported by the Estonian Centre of Behavioural and Health Sciences and Estonian Science Foundation grants no. 3277, 5209 and 6788. J. R. Ruiz and F. B. Ortega were supported by a grant from the Ministerio de Educación y Ciencia de España (AP2003-2128, AP2004-2745), and CSD grants 05/UPB32/0, 109/UPB31/03 and 13/UPB20/04. T. Veidebaum was supported by grants from the Ministry of Education and Science (0942706s06 and 0192112s02).

Duality of interest None of the authors has any conflict of interest.

\section{References}

1. Cruz ML, Shaibi GQ, Weigensberg MJ, Spruijt-Metz D, Ball GD, Goran MI (2005) Pediatric obesity and insulin resistance: chronic disease risk and implications for treatment and prevention beyond body weight modification. Annu Rev Nutr 25:435-468

2. Srinivasan SR, Myers L, Berenson GS (2002) Predictability of childhood adiposity and insulin for developing insulin resistance syndrome (syndrome $\mathrm{X}$ ) in young adulthood: the Bogalusa Heart Study. Diabetes 51:204-209

3. Ruiz JR, Rizzo NS, Hurtig-Wennlöf A, Ortega FB, Wärnberg J, Sjöström M (2006) Relations of total physical activity and intensity to fitness and fatness in children: the European Youth Heart Study. Am J Clin Nutr 84:299-303

4. Gutin B, Yin Z, Humphries MC, Barbeau P (2005) Relations of moderate and vigorous physical activity to fitness and fatness in adolescents. Am J Clin Nutr 81:746-750

5. Ortega FB, Tresaco B, Ruiz JR et al (2007) Cardiorespiratory fitness is associated with favorable abdominal adiposity in adolescents. Obesity (in press)
6. Gutin B, Yin Z, Humphries MC, Hoffman WH, Gower B, Barbeau P (2004) Relations of fatness and fitness to fasting insulin in black and white adolescents. J Pediatr 145:737-743

7. Imperatore G, Cheng YJ, Williams DE, Fulton J, Gregg EW (2006) Physical activity, cardiovascular fitness, and insulin sensitivity among US adolescents: the National Health and Nutrition Examination Survey, 1999-2002. Diabetes Care 29:1567-1572

8. Ruiz JR, Ortega FB, Meusel D, Harro M, Oja P, Sjöström M (2006) Cardiorespiratory fitness is associated with features of metabolic risk factors in children. Should cardiorespiratory fitness be assessed in a European health monitoring system? The European Youth Heart Study. J Public Health 14:94-102

9. Ball GD, Shaibi GQ, Cruz ML, Watkins MP, Weigensberg MJ, Goran MI (2004) Insulin sensitivity, cardiorespiratory fitness, and physical activity in overweight Hispanic youth. Obes Res 12:77-85

10. Shaibi GQ, Ball GD, Cruz ML, Weigensberg MJ, Salem GJ, Goran MI (2006) Cardiovascular fitness and physical activity in children with and without impaired glucose tolerance. Int J Obes (Lond) 30:45-49

11. Wennlöf AH, Yngve A, Sjöström M (2003) Sampling procedure, participation rates and representativeness in the Swedish part of the European Youth Heart Study (EYHS). Public Health Nutr 6:291-299

12. Poortvliet E, Yngve A, Ekelund U et al (2003) The European Youth Heart Survey (EYHS): an international study that addresses the multidimensional issues of CVD risk factors. Forum Nutr 56:254-256

13. Lohman TG, Roche AF, Martorell R (1991) Anthropometric standardization reference manual. Human Kinetics, Champaign, IL

14. Rennie KL, Livingstone MB, Wells JC et al (2005) Association of physical activity with body-composition indexes in children aged 6-8 y at varied risk of obesity. Am J Clin Nutr 82:13-20

15. Tanner JM, Whitehouse RH (1962) Standards for subcutaneous fat in British children. Percentiles for thickness of skinfolds over triceps and below scapula. BMJ 1:446-450

16. Matthews DR, Hosker JP, Rudenski AS, Naylor BA, Treacher DF, Turner RC (1985) Homeostasis model assessment: insulin resistance and beta-cell function from fasting plasma glucose and insulin concentrations in man. Diabetologia 28:412-419

17. Wennlöf AH, Yngve A, Nilsson TK, Sjöström M (2005) Serum lipids, glucose and insulin levels in healthy schoolchildren aged 9 and 15 years from Central Sweden: reference values in relation to biological, social and lifestyle factors. Scand J Clin Lab Invest 65:65-76

18. Hansen HS, Froberg K, Nielsen JR, Hyldebrandt N (1989) A new approach to assessing maximal aerobic power in children: the Odense School Child Study. Eur J Appl Physiol Occup Physiol 58:618-624

19. Riddoch C, Edwards D, Page A et al (2005) European Youth Heart Study - cardiovascular disease risk factors in children: rationale, aims, study design, and validation of methods. J Phys Act Health 2:115-129

20. Holm S (1979) A simple sequentially rejective multiple test procedure. Scand J Statist 6:65-70

21. Caprio S, Hyman LD, Limb C et al (1995) Central adiposity and its metabolic correlates in obese adolescent girls. Am J Physiol 269:E118-E126

22. Owens S, Gutin B, Barbeau P et al (2000) Visceral adipose tissue and markers of the insulin resistance syndrome in obese black and white teenagers. Obes Res 8:287-293

23. Goran MI, Bergman RN, Gower BA (2001) Influence of total vs visceral fat on insulin action and secretion in African American and white children. Obes Res 9:423-431

24. Taylor RW, Jones IE, Williams SM, Goulding A (2000) Evaluation of waist circumference, waist-to-hip ratio, and the conicity index as screening tools for high trunk fat mass, as measured by dual-energy X-ray absorptiometry, in children aged 3-19 y. Am J Clin Nutr 72:490-495

25. Brambilla P, Bedogni G, Moreno LA et al (2006) Crossvalidation of anthropometry against magnetic resonance imaging for the 
assessment of visceral and subcutaneous adipose tissue in children. Int J Obes (Lond) 30:23-30

26. van der Kooy K, Seidell JC (1993) Techniques for the measurement of visceral fat: a practical guide. Int J Obes Relat Metab Disord 17:187-196

27. Donahue RP, Prineas RJ, DeCarlo Donahue R, Bean JA, Skyler JS (1996) The female 'insulin advantage' in a biracial cohort: results from the Miami Community Health Study. Int J Obes Relat Metab Disord 20:76-82

28. Munoz J, Derstine A, Gower BA (2002) Fat distribution and insulin sensitivity in postmenopausal women: influence of hormone replacement. Obes Res 10:424-431

29. Ortega FB, Ruiz JR, Castillo MJ et al (2005) Low level of physical fitness in Spanish adolescents. Relevance for future cardiovascular health (AVENA study). Rev Esp Cardiol 58:898-909

30. Moreno LA, Mesana MI, Gonzalez-Gross M et al (2006) Anthropometric body fat composition reference values in Spanish adolescents. The AVENA Study. Eur J Clin Nutr 60:191-196

31. Ford ES, Li C, Imperatore G, Cook S (2006) Age, sex, and ethnic variations in serum insulin concentrations among US youth: findings from the National Health and Nutrition Examination Survey 1999-2002. Diabetes Care 29:2605-2611

32. Bunt JC, Salbe AD, Harper IT, Hanson RL, Tataranni PA (2003) Weight, adiposity, and physical activity as determinants of an insulin sensitivity index in Pima Indian children. Diabetes Care 26:2524-2530

33. Ignico AA, Mahon AD (1995) The effects of a physical fitness program on low-fit children. Res Q Exerc Sport 66:85-90

34. Mahon AD, Vaccaro P (1994) Cardiovascular adaptations in 8- to 12-year-old boys following a 14-week running program. Can J Appl Physiol 19:139-150

35. Brage S, Wedderkopp N, Ekelund U et al (2004) Objectively measured physical activity correlates with indices of insulin resistance in Danish children. The European Youth Heart Study (EYHS). Int J Obes Relat Metab Disord 28:1503-1508

36. Platat C, Wagner A, Klumpp T, Schweitzer B, Simon C (2006) Relationships of physical activity with metabolic syndrome features and low-grade inflammation in adolescents. Diabetologia 49:2078-2085

37. Andersen LB, Boreham CA, Young IS et al (2006) Insulin sensitivity and clustering of coronary heart disease risk factors in young adults. The Northern Ireland Young Hearts Study. Prev Med 42:73-77

38. Le Stunff C, Fallin D, Schork NJ, Bougneres P (2000) The insulin gene VNTR is associated with fasting insulin levels and development of juvenile obesity. Nat Genet 26:444-446

39. Wallace TM, Levy JC, Matthews DR (2004) Use and abuse of HOMA modeling. Diabetes Care 27:1487-1495 